\title{
Writing a Research Article. Are We Ignoring the Basics?
}

\author{
Debajyoti Mohanty • Naveen Sharma • \\ Bhupendra Kumar Jain
}

Received: 22 March 2013 / Accepted: 1 July 2013 / Published online: 24 July 2013

(C) Association of Surgeons of India 2013

We have read the article 'Comparative study between staplers and conventional (hand-sewn) anastomosis in gastrointestinal surgery' by Bangaru et al. [1] with interest. The authors had evaluated the two techniques in three different types of surgical procedures with 40 patients assigned to each procedure group.

At this point, the authors had only mentioned of random allocation of patients in each group into the two treatment arms comprising of stapler and hand-sewn anastomosis, respectively. A more detailed description of the technique of randomization and the method of blinding employed would have enlightened the readers about the study protocol. The table for inclusion criteria is showing indications for the different procedures and the number of patients undergoing each procedure instead of highlighting the basis on which the patients were selected for the study. Defining the primary outcome measure of a study is important from the point of view of sample size calculation. The present study had listed total operating time, time to return of bowel sounds, day of resumption of oral feeds, postoperative hospital stay and anastomotic leak as the outcome parameters without categorizing them into primary and secondary outcome measures; thus, the basis on which the sample size of 120 patients was calculated is not clear to us.

The method, result and discussion sections in this article did not strictly adhere to their intended purpose as data interpretation was carried out in all the three sections. A table

D. Mohanty $\cdot$ N. Sharma $(\bowtie)$

Department of Surgery, UCMS, 143, Sreshtha Vihar, 110092 Delhi,

India

e-mail: drnsemail@gmail.com

D. Mohanty

e-mail: debajyoti.mohanty@gmail.com

B. K. Jain

Department of Surgery, UCMS and GTB Hospital, Delhi, India

e-mail: bhupendrakjain@gmail.com showing baseline characteristics of included patients is mandatory when presenting data in a comparative study. Description of data has two components: central tendency (mean, median, mode) and dispersion (standard deviation, interquartile range). The authors had reported all their data in the form of mean value only without mentioning degree of dispersion. This incomplete information does not allow further statistical analysis to be contemplated including calculation of the unpaired $t$ test as mentioned by the authors in the abstract section only. Figures and graphs should be self-explanatory and easily interpreted by the readers. The graphs provided in this article did not mention the units of measurement in both the abscissa and ordinate.

The discussion section should explain any significant difference observed in the outcome measures and its relevance in the light of similar other existing studies rather than merely reporting the results and its concordance to published literature. Likewise, it will be interesting to know the reasons for delayed discharge of patients following colorectal anastomosis even though they were started early on oral feeds than the gastrojejunostomy group. The need for a long follow-up period of 2-22 months should also be clarified as the last outcome parameter (anastomotic leak) can always be detected in the immediate postoperative period.

Lastly, we would like to appeal to all the contributing authors that devoting a little extra effort and attention to correct presentation of data will supplement their hard and painstaking research work and will help to achieve the long-cherished goal of establishing IJS as a leading global surgical journal.

\section{Reference}

1. Bangaru H, Veitla RMR, Pigilam M, Kunwargiri GK (2012) Comparative study between staplers and conventional (hand-sewn) anastomosis in gastrointestinal surgery. Indian J Surg 74(6):462-467 